\title{
Nanoscale imaging approaches to quantifying the electrical properties of
}

\section{pathogenic bacteria}

\author{
Ryan Berthelot ${ }^{1}$ and Suresh Neethirajan ${ }^{1 *}$ \\ ${ }^{1}$ BioNano Laboratory, Engineering Department, University of Guelph, 50 Stone Rd. E, Guelph, Canada \\ *Correspondence: S. Neethirajan Email: sneethir@uoguelph.ca
}

\section{Abstract}

Biofilms are natural, resilient films formed when microorganisms adhere to a surface and form a complex three-dimensional structure that allows them to persist in a wide variety of environments. Readily forming in hospitals and on medical equipment, biofilms are frequent causes of infections and their subsequent complications. Due to the complexity of these structures, systematically studying individual bacterial cells and their interactions with their surrounding environment will provide a deeper understanding of the processes occurring within the biofilm as whole versus bulk population based methods that do not differentiate individual cells or species. Methods based on atomic force microscopy (AFM) are particularly suited to the study of individual cells, but are underutilized for the study of bacterial electrical properties. The ability of electrical currents to impair bacterial attachment is well documented, but to utilize electrical current as an effective antibacterial treatment, it is important to understand the electrical properties of bacteria. Therefore, we used AFM, Kelvin probe force microscopy, and ResiScope to measure the surface potential and conductance of Psuedomonas aeruginosa and methicillin resistance Staphylococcus aureus (MRSA) on gold and stainless steel. This is the first study to directly measure the electrical resistance of single bacterial cells using ResiScope. Our goal was to develop a framework for measuring biological molecules using conductive atomic force microscopy. We found that the 
24 average resistance for $P$. aeruginosa was $135.4 \pm 25.04 \mathrm{G} \Omega$, while MRSA had an average of

$25173.4 \pm 16.28 \mathrm{G} \Omega$. Using KPFM, the surface potential of MRSA shifted from $-0.304 \mathrm{~V}$ to $0.153 \mathrm{~V}$

26 and from $-0.280 \mathrm{~V}$ to $0.172 \mathrm{~V}$ for $P$. aeruginosa on gold versus stainless steel substrates,

27 respectively. In an attempt to identify a potential charge carrier, peptidoglycan was also measured

28 with the ResiScope module and shown to have a resistance of $105 \mathrm{G} \Omega$.

29 Keywords: Resiscope; bacteria; conductive AFM; nanoscale imaging; kelvin probe force 30 microscopy

\section{Introduction}

The World Health Organization (WHO) has deemed antimicrobial resistance a cardinal

34 threat facing humanity [1]. A report from the Centre for Disease Control (CDC), published in 2015

35 underscores this threat, estimating that approximately one in every 100 individuals infected with

36 drug resistant microbes will perish from such an infection [2]. This has caused a great deal of

37 interest in the development of alternate preventative and treatment methods effective against

38 resistance species of pathogenic bacteria, such as Pseudomonas aeruginosa and Methicillin-

39 resistant Staphylococcus aureus (MRSA).

Not only do bacteria gain resistance due to the heavy and widespread use of antimicrobials

41 in medicine, cleaning products, soaps, and sanitizers [1], but specialized communities of bacteria

42 cells, known as biofilms, are associated with inherent antimicrobial resistance and recalcitrance to

43 removal. Biofilms can easily proliferate within the human body, especially at wound sites where

44 the protective epithelium is compromised. Any biofilm infection, if not cleared, can be life-

45 threatening. Biofilms are initialized when bacteria adhere to a surface and begin to accumulate and

46 produce an extracellular matrix, which enmeshes and protects these organisms, while increasing 
47 their adhesion strength. While antimicrobials are effective at eliminating the outer layers of

48 bacteria near the surface of the biofilm, the bottom layer, which is tightly bound to the substrate

49 and protected by the surrounding biomass, can persist and continue to proliferate $[3,4]$.

50 MRSA and $P$. aeruginosa are opportunistic pathogens that form biofilms and are

51 commonly associated with nosocomial infections [5]. P. aeruginosa is a Gram-negative, rod-

52 shaped bacterium that is highly proficient in forming biofilms. The ability of P. aeruginosa to

53 attach and form biofilms on a range of metallic and non-organics surfaces, has caused serious

54 issues in hospitals (e.g. catheter infections in immunocompromised individuals) [6]. P. aeruginosa

55 is also responsible for complications in the treatment of illnesses that affect the respiratory system,

56 such as pneumonia or cystic fibrosis. Once inside the lungs, $P$. aeruginosa excretes a "slime"

57 matrix composed of various biological components that can affect cilia movement and cause

58 inflammation within the lungs, thus causing breathing complications [6]. This biofilm forming

59 ability makes $P$. aeruginosa especially dangerous when mixed with other bacteria, as biofilms can

60 turn a surface that is non-ideal for bacterial attachment into a surface to which other bacteria, such

61 as MRSA, will preferentially bind [5]. A single microbe attaching to a surface, can initiate a chain

62 reaction, creating an environment and surface more prone to infection of additional foreign,

63 pathogenic species, such as other bacterial strains, fungi and viruses [7,8]. MRSA is Gram-positive

64 cocci that once attached to a surface, secretes adhesins and toxins, facilitating attachment, all while

65 damaging the surrounding human cells and tissue [9]. Therefore, these two organisms are

66 particularly threatening to human health and require unique strategies to prevent, manage, and treat

67 infection.

68 In terms of creating antibacterial treatments that target bacterial biofilms, electricity has

69 shown great potential in disrupting bacterial adherence. The utilization of electricity as an 
70 antimicrobial therapy has been investigated not only because it can reduce bacterial attachment,

71 but it may also accelerate the wound healing process, serving two essential functions in wound

72 healing therapy [10-12]. Cathodic current across conductive surfaces can reduce attachment by up

73 to $80 \%$, potentially leading to the complete detachment of adherent bacteria [10]. Furthermore,

74 alternating current in wound healing applications on guinea pigs, speeds healing times when

75 directly applied to incised regions on the skin [13]. Therefore, researchers have begun creating

76 devices that allow for the application of electricity to a wound site. For example, Park et al. created

77 a polyester material coated with small, alternating $\mathrm{Zn}$ and Ag dots placed in an array across the

78 surface. The difference in electrical potentials between the dots caused microcurrents to be

79 generated across the wound site, affecting bacterial migration [14]. This is based on the principle

80 that under the influence of an electrical current, the motility of bacteria, such as $P$. aeruginosa and

81 Escherichia coli is impaired or guided in a specific direction via electrotaxis [15]. Although the

82 electrical inhibition of bacterial attachment has been well documented, we do not fully understand

83 the electrical properties of bacteria and how this influences bacterial behavior.

New state-of-the-art methods are being applied to characterize the surfaces charges of

85 bacteria and the impact of current on bacteria. Among these, AFM is a nanoscale imaging

86 technique that measures the topography of a surface. It has tremendous advantages over other

87 nano-imaging techniques such as scanning electron microscopy, in that it does not require drastic

88 changes in the environment (e.g. changes in pressure or temperature) to successfully image a

89 sample [16]. ResiScope is a module for AFM, in the category of conductive AFM (CAFM) that

90 simultaneously measures the current and resistance of a sample [17]. Similarly, Kelvin probe force

91 microscopy (KPFM) is a conductive mode of AFM that simultaneously measures the contact

92 potential difference between the tip and the sample. This value is a measure of the surface potential 
93 [18]. This method of simultaneous acquisition provides a tremendous advantage in relating the

94 electrical properties of a sample to its physical structure. In this study, we utilized AFM, KPFM

95 and the ResiScope to simultaneously acquire topography, surface potential, and current to measure

96 the electrical resistance and surface potential of single MRSA and P. aeruginosa cells. Our goal

97 was to develop a framework for measuring biological molecules using conductive atomic force

98 microscopy (AFM), measures that will help us to understand how electrical current impacts

99 pathogenic bacteria.

\section{2. Methods}

\subsection{Bacterial strains and culture conditions}

103 Strains of Pseudomonas aeruginosa and Methicillin-resistant Staphylococcus aureus (MRSA)

104 were obtained from Dr. Scott Weese and Joyce Rousseau of the Pathobiology Department at the

105 University of Guelph. Strains were streaked and cultured on 5\% blood agar plates and grown at

$10637^{\circ} \mathrm{C}$ for 24 hours before use. Individual colonies were chosen and transferred into $6 \mathrm{~mL}$ of tryptic

107 soy broth (TSB) solution to culture at $37^{\circ} \mathrm{C}$ in a shake incubator rotated at 200 rpm for 24 hours.

108 One $\mathrm{mL}$ of the bacterial broth was then transferred to $1.5 \mathrm{~mL}$ microfuge containers and centrifuged

109 at $1000 \mathrm{xg}$ for 5 minutes. The excess liquid was then decanted, and the remaining cells were

110 resuspended into $1 \mathrm{~mL}$ of deionized water. This rinsing processes was repeated twice.

\subsection{Sample preparation for AFM/KPFM/ResiScope imaging}

113 Gold covered stainless steel discs, stainless steel discs, and sputter-coated gold mica substrates

$114(\sim 100 \mathrm{~nm}$ coating) were used in these experiments as conductive interfaces. Clean, gold and

115 stainless steel discs were sonicated for 1 minute in de-ionized water to remove excess waste from 
116 the surface. Substrates were then rinsed with $1 \mathrm{~mL}$ of deionized water and left to dry for 4 hours.

117 Four-hundred $\mu \mathrm{L}$ of the resuspended, rinsed bacterial solution was placed onto the stainless steel

118 and the Au-coated mica, while $250 \mu \mathrm{L}$ were deposited onto the gold discs (amount chosen to

119 maximize surface coverage for each sample). Substrates were then incubated at room temperature

120 for 20 minutes, allowing for bacterial attachment. This length of time produces highly dispersed

121 populations for single cell imaging on the atomic force microscope. The excess solution on the

122 substrates was then gently rinsed with $1 \mathrm{~mL}$ of deionized water using a micropipette. Substrates

123 were allowed to dry overnight before imaging.

124

\subsection{Preparation of Au-Coated mica}

126 Mica sheets were cut into approximately 1.8 x $1.8 \mathrm{~cm}$ squares using sharp scissors. Individual 127 sheets were cleaved to ensure a clean, flat surface with no flaking. Mica sheets were then sputter128 coated under vacuum with $\sim 100 \mathrm{~nm}$ of gold. This thickness allows for high current application in 129 measurements without the degradation of the thin metal film.

\subsection{Imaging AFM/KPFM/Resiscope}

132 All AFM imaging was done on the Agilent 5500 series AFM. The ResiScope module from

133 Concept Scientific Instruments was used to make current and resistance measurements.

134 The conversion of resistance imaging units in Volts to Ohms is given by $R=10^{(V+2)}$. All

135 ResiScope images were taken with a potential of $0.7 \mathrm{~V}$ applied to the conductive substrate.

136 Conductivity imaging was done using conductive PtIr-coated Si tips of $75 \mathrm{kHz}, 2 \mathrm{~N} / \mathrm{m}$ and $225 \mathrm{~nm}$

137 in length. KPFM measurements were taken using Mikromasch Pt coated cantilevers of $75 \mathrm{kHz}$,

$1382 \mathrm{~N} / \mathrm{m}$ and $240 \mathrm{~nm}$. Measured Cells were chosen based upon the lack of surrounding, noticeable 
139 contamination (e.g. EPS), as well as their total exposed circumference. Single cells were targeted

140 for measurement in this experiment, leaving roughly at least half of the total cell circumference

141 exposed.

$142 \quad 2.5$ Raman Spectroscopy

143 All Raman measurements were done on the Advantage series near-infrared Raman spectrometer

144 by DeltaNu. A wavelength of $785 \mathrm{~nm}$ was used to collect spectra. Three centrifuged, rinsed 145 quantities of MRSA and P. aeruginosa were combined and resuspended in deionized water to 146 ensure a high concentration of cells. Twenty $\mu \mathrm{L}$ of rinsed bacterial solution was then deposited 147 onto gold SERS substrates from Ocean Optics and left to dry for a period of three days. Purified 148 peptidoglycan samples from Staphylococcus aureus were bought from Sigma-Aldrich. A 4 mg/mL 149 solution of peptidoglycan was left to settle for 1 minute. Twenty-five $\mu \mathrm{L}$ of the separated and 150 suspended peptidoglycan sample was then pipetted onto the SERS substrate and left to dry for 151 three days.

\section{Results}

1543.1 Bacterial resistivity and potential measurements

156 relevance. Conductive measurements were made on uncolonized bare and gold-coated stainless

157 steel and on the same surfaces after $P$. aeruginosa and MRSA were allowed to adhere. Due to the 158 roughness of the stainless steel, along with its increased reactivity (versus gold), we were 159 unsuccessful in taking measurements on this surface using the ResiScope. However, we were able 160 to acquire CAFM images of MRSA and P. aeruginosa on gold-coated surfaces (Figure 1). Table 1611 shows the average resistance for bacterial cells adhered to the surface. The average resistance 
for $P$. aeruginosa was $135 \pm 25 \mathrm{G} \Omega$, while MRSA had an average of $173 \pm 16 \mathrm{G} \Omega$. The two values were not significantly different from one another. However, we may see differences with a larger sample size, as the average is based on resistance values from an average of four cells. These measurements are in alignment with those obtained in another study that quantified the resistance of $E$. coli cells suspended across two, conductive gold interdigitated fingers; results resistance 167 values were $\sim 396 \mathrm{G} \Omega[19]$.

We also acquired KPFM measurements of MRSA and $P$. aeruginosa on stainless steel and gold-coated substrates to gain a better understanding of the relationship between surface potential and potential charge transfer (Figure 2, Table 1). Both of the bacterial cell surface potentials switched from negative to positive between the gold and stainless steel substrates. MRSA had a surface potential of $-0.304 \mathrm{~V}$ for gold and $0.153 \mathrm{~V}$ for stainless steel, and $P$. aeruginosa $-0.280 \mathrm{~V}$

173 for gold and $0.172 \mathrm{~V}$ for stainless steel. A similar effect was reported by Birkenhauer et al., where

174 the surface potential of MRSA cells switched from positive on stainless steel to negative on gold.

175 This suggests that surface potential varies across bacterial strains and is influenced by the surface 176 to which the cells bind [5]. Finally, in an attempt to measure charge transfer, a 500 x $500 \mathrm{~nm}$ close177 up resistance image of $P$. aeruginosa on gold was acquired (Figure 3). KPFM close-ups emphasize 178 the difference in surface potential at the cell-gold interface.

\subsection{The role of peptidoglycan in potential measures}

We went on to investigate which bacterial substances might be involved in forming the conductive path shown in Figure 1. In solution, bacteria are conductive due to the functions of their ion channels. However, their outer membranes are largely composed of polysaccharides and

184 lipids, which are non-conductive. Based on the structural properties of the membranes in MRSA and $P$. aeruginosa, the bulk of the electrical measurements would be made while in contact with 
the outer peptidoglycan layer. Therefore, we dried a $4 \mathrm{mg} / \mathrm{mL}$ of peptidoglycan on gold coated mica for 24 hours at $37^{\circ} \mathrm{C}$ to image the electrically conductive properties of peptidoglycan to determine if its resistive properties correlate with images of bacteria taken earlier. We found micrometer sized islands of peptidoglycan had formed, with a height of approximately $40 \mathrm{~nm}$ in height (Figure 4). To confirm that the purified peptidoglycan was indeed peptidoglycan, Raman spectroscopy was used, and the results were correlated with scans of $P$. aeruginosa and MRSA

192 that show it is present in both samples (Figure 5). The peak at $\sim 730 \mathrm{~nm}^{-1}$ is attributed to the 193 stretching of the glycosidic rings in the NAG and NAM molecules present in the peptidoglycan 194 layer surrounding the cells [19-20]. Relative intensity measures show a smaller peak for $P$. aeruginosa, most likely due to the thinner layer of peptidoglycan and its partial obstruction by

196 lipopolysaccharides. This confirms that the molecule being measured on the surface is in fact 197 peptidoglycan. CAFM measurements of peptidoglycan on the gold sputter coated mica show an average resistance value of $9.021 \mathrm{~V}$ or $105 \mathrm{G} \Omega$ (Figure 6). This resistance value will change depending of the thickness of the peptidoglycan sample.

\section{Discussions}

AFM, KPFM, and ResiScope are excellent tools for studying the electrical potential of

203 bacteria, as demonstrated by our results. However, the conditions must be carefully controlled to

204 ensure ideal measurement. Controlling the quality of a ResiScope image can be accomplished by 205 varying the force setpoint and the potential applied on the substrate. By increasing the setpoint and 206 increasing the tip force exerted upon the sample, increased contact improves the consistency of the 207 conductance measurements. However, if the force is raised too high, it can wear the conductive 208 coating off the tip. Another option to produce a better electrical signal is to increase the potential 
applied to the substrate, thus raising the flow of current from the substrate to the tip. However,

210 raising the voltage too high can increase the reactivity between the tip and the sample, leading to

211 degradation of the thin metal. For these reasons, the choice of tip becomes critical [17]. Originally,

212 we used the same platinum (Pt) tips for CAFM and KPFM. However, the Pt tips eroded rapidly,

213 wearing away the metal coating. For these reasons, PtIr tips were used, which increased the signal

214 response by providing a more stable flow of electrons [21]. PtIr contact mode tips with a low spring

215 constant of $0.1 \mathrm{~N} / \mathrm{m}$ are superior to Pt tips, but still generate a significant amount of noise. After

216 testing PtIr tips with a midrange spring constant of $3 \mathrm{~N} / \mathrm{m}$ and $75 \mathrm{kHz}$, we found these were ideal

217 for our task. The higher spring constant allowed for more consistent contact between the tip and

218 the surface. This combination gave the best and most consistent measurements for both biological

219 and test samples. Our experiences underscore the importance of carefully selecting best settings

220 and tips for CAFM analysis; we found that until a method is established for a particular sample

221 type, this is likely to be a trial and error process. Our work establishes a set a parameters that can

222 be used as a starting point for subsequent studies of a similar nature.

As for CAFM, we found that there are a variety of parameters that influence the success of

224 ResiScope analysis. The version of ResiScope currently used by our laboratory is limited to contact

225 mode. Contact mode, especially when used for inorganic samples, allows for better electrical

226 contact between the tip and the substrate, which leads to more stable measurements. However,

227 there are some shortcomings to using contact mode for these studies. First, it can easily dislodge a

228 sample from the surface if it is weakly bound. Second, the high friction force of the cantilever

229 pressing onto the metallic surface quickly erodes the thin film of conductive material deposited

230 onto the Si tips [16]. There is a potential for combining KPFM and ResiScope images to study

231 charge transfer, as a result of differences in the localized potential between the cell and the 
232 substrate. Our results give a reasonable starting point for such studies, but additional optimization

233 is necessary to improve image quality for our application.

Our results and experiences demonstrate that there are a variety of options for enhancing

235 and optimizing the measurement of bacterial electrical properties using CAFM. The experiments

236 in this study provide a framework for future analysis and highlight several pathways for four

237 ongoing research. For example, one direction would be to study bacteria in a more hydrated

238 environment, similar to their natural habitat. Although, electrical measurements cannot be made

239 in liquid, an environment humid enough to maintain the cells in a moist climate could restore the

240 function of bacterial ion channels, while being conducive to CAFM, thereby allowing for the

241 measurement of their charge transfer properties. Although multi-drug resistant strains of bacteria

242 are highly relevant to the end application of this project, measuring a known conductive bacterium,

243 such as G. sulfurreducens, may also help in the development and optimization CAFM techniques

244 to be later be applied to less conductive strains. Furthermore, strains of G. sulfurreducens form

245 conductive biofilms [22-23], offering the potential for charge transference and propagation to be

246 studied in these much larger structures.

247 As denoted earlier, MRSA and P. aeruginosa strains were initially chosen for electrical

248 measurement, because they are two common opportunist pathogens that readily cause wound

249 infection. Wound infections represent a prime area where electrical current could be

250 therapeutically applied as it may help to remove bacteria, while accelerating wound healing. In

251 addition to comparing potential conductive differences between Gram-positive and Gram-negative

252 bacteria, the motile nature of $P$. aeruginosa allows for the imaging and investigation of the

253 electrical properties of pili and flagella. We plan to investigate this area further in future studies

254 and to apply these techniques to the study of other interesting or relevant bacteria. For example, 
255 CAFM has direct measurement capabilities that could potentially help to elucidate how $G$.

256 sulfurreducens pili exhibit metallic-like conductivity [24-25].

257 It is not entirely clear which component(s) of the bacteria studied here are responsible for

258 conducting the charge. If peptidoglycan is acting as the charge carrier, it is somewhat surprising

259 then that $P$. aeruginosa does not display a higher resistivity, as it is covered by an outer layer of

260 lipopolysaccharides. This suggests that an alternate conductive structure may be responsible for

261 conductance observed in this study. This is something that we could investigate in future studies,

262 as we have several parameters worked out for examining external bacterial structures using

263 ResiScope. For the measurement of peptidoglycan, we switched the substrate from gold-coated

264 stainless steel to gold sputter-coated mica, because the high surface roughness of the original

265 substrates could potentially have made it difficult to distinguish the peptidoglycan from the surface

266 itself. Additionally, we determined that the gold-sputtered mica represents a much cleaner sample

267 that is less easily contaminated prior to imaging. Although residual material is washed off the gold-

268 coated stainless steel, the high surface roughness will retain a certain degree of contaminants from

269 the environment that can obstruct electrical measurements. Conversely, the cleaved mica provides

270 a pristine surface, ensuring that the only thing being measured is the purified peptidoglycan

271 sample. Direct measurements of dried bacterial cells are seldom collected; most electrical

272 measurements of bacteria focus on the planktonic state or individual live cells in solution [26]. Our

273 work provides a framework for the future measurement of bacterial conductance in individual

274 bacterial cells. 


\section{Conclusions}

Our study successfully establishes a novel methodology for the imaging and analysis of the conductance of single bacterial cells from medically relevant bacteria, such as $P$. aeruginosa and MRSA. Our method is adaptable and can be modified as necessary to study the conductance of other bacterial species or biological substances. The average resistance values obtained in our study are comparable to those obtained in a prior study of E. coli, helping to validate our technique. Our work opens the door to further investigation of the electrical properties of pathogenic bacteria, information that will be essential in developing electroceuticals. Such approaches would be a great benefit for wound healing, as early studies suggest that the application of an electrical current can help to eliminate potential pathogens, while accelerating wound healing. However, to optimize such treatments, a better understanding of the localized electrical properties of adherent bacteria is necessary. Our work helps to establish a "toolbox" for investigating the electrical properties of an

290 array of bacterial species.

\section{Acknowledgements}

Financial support for this research was provided by the Natural Sciences and Engineering

294 Council of Canada (Grant\#400705) and the Ontario Ministry of Research and Innovation (Grant 295 \#300142). The authors would like to thank Dr. Scott Weese and Ms Joyce Rousseau of the 296 Pathobiology Department of the University of Guelph for providing bacterial strains as clinical 297 samples from canine patients. 


\section{References}

1) World Health Organization 2015 Worldwide country situation analysis: response to antimicrobial resistance. Retrieved from http://apps.who.int/iris/bitstream/10665/163468/1/9789241564946_eng.pdf?ua=1\&ua=1

2) Centers for Disease Control and Prevention 2013 Antibiotic Resistance Threats in the United States. U.S. Department of Health and Human Services:1600 Clifton Road Atlanta, GA 303294027 USA

3) Neethirajan S and Clond M 2014 Medical Biofilms-Nanotechnology Approaches J. Biomed. Nanotechnology. 10 1-22

4) Donlan R M 2002 Biofilms: microbial life on surfaces Emerg. Infect. Dis. 8(9) 881-890

5) Birkenhauer E and Neethirajan S 2014 Characterization of electrical surface properties of monoand co-cultures of Pseudomonas aeruginosa and methicillin-resistant Staphylococcus aureus using Kelvin probe force microscopy $R S C A d v .442432-42440$

6) Rehm B 2008 Pseudomonas: model organism, pathogen, cell factory, Weinheim, Wiley-VCH

7) Bertesteanu S, Triaridis S, Stankovic M, Lazar V, Chifiriuc M C, Vlad M and Grigore R 2014 Polymicrobial wound infections: Pathophysiology and current therapeutic approaches Int. J. Pharm. 463 119-126

8) Harmsen M, Yang L, Pamp S J, and Tolker-Nielsen T 2010 An update on Pseudomonas aeruginosa biofilm formation, tolerance, and dispersal FEMS. Immunol. Med. Mic. 59 253-268

9) Ofek I 1994 Bacterial adhesion to animal cells and tissues, New York, Chapman \& Hall 10) Hong H S, Jeong J, Shim S, Kang H, Kwon S, Ahn K and Yoon J 2008 Effect of Electric Currents on Bacterial Detachment and Inactivation Biotechnol. Bioeng. 100 379-386

11) Borden A J, Mei H C, Busscher H J 2005 Electric block current induced detachment from surgical stainless steel and decreased viability of Staphylococcus epidermidis Biomaterials. 26 6731-6735

12) Kerr A, Hodgkiess T, Cowling M J, Beveridge C M, Smith M J and Parr A C S 1998 A Novel Technique to Prevent Bacterial Fouling, Using Imposed Surface Potential J. App. Microbiol. 85 1067-1072

13) Mehmandoust F G, Torkaman G, Firoozabadi M and Talebi G 2007 Anodal and Cathodal Pulsed Electrical Stimulation on Skin Wound Healing in Guinea Pigs J. Rehabil. Res. Dev. 44 611618 
14) Park S, Kim H, Makin I, Skiba J and Izadjoo M 2015 Measurement of Microelectric potentials in a Bioelectrically-Active Wound Care Device in the Presence of Bacteria J. Wound. Care. 24 23-33

15) Shi W, Lentz M J, Adler J 1996 Effect of the Surface Composition of Motile Escherichia coli and motile Salmonella Species on the Direction of Galvanotaxis J. Bacteriol. 178 1113-1119

16) Eaton P J and West P 2010 Atomic force microscopy. New York, Oxford University Press

17) Resiscope User Guide 2011 R10A. Plateau de Saclay, France: Concept Scientific Instruments

18) Melitz W, Shen J, Kummel A C and Lee S 2011 Kelvin probe force microscopy and its application Surf. Sci. Rep. 66 1-27

19) Lu Y, Chuang Y, Chen Y, Shu A, Hsu H, Chang H and Yew T 2008 Bacteria Detection Utilizing Electrical Conductivity Biosens. Bioelectron. 23 1856-1861

20) Jarvis R M, Brooker A and Goodacre R 2006 Surface-enhanced Raman scattering for the rapid discrimination of bacteria Faraday. Discuss. R. Soc. Chem. 132 281-292

21) Jamond N, Chretien P, Houze F, Lu L, Largeau L, Mauain O, Travers L, Harmand J C, Glas F, Lefeuvre E, Tchernycheva M and Gogneau N 2016 Piezo-generator integrating a vertical array of GaN nanowires Nanotechnology. 27 325403-325412

22) Malvankar N S and Lovley D R 2014 Microbial nanowires for bioenergy applications Curr. Opin. Biotech. 27 88-95

23) Malvankar N S, Tuominen M T and Lovley D R 2012 Lack of cytochrome involvement in long-range electron transport through conductive biofilms and nanowires of Geobacter sulfurreducens Energ. Environ. Sci. 9 8651-8659

24) Veazey J P, Reguera G and Tessmer S H 2011 Electronic properties of conductive pili of the metal-reducing bacterium Geobacter sulfurreducens probed by scanning tunneling microscopy Phys. Rev. E. 84 060901-1-4

25) Reguera G and McCarthy K 2005 Extracellular Electron Transfer via Microbial Nanowires Nature. 435 1098-1101

26) Mansor M A, Ahmad M R 2015 Single Cell Electrical Characterization Techniques Int. J. Mol. Sci. 16 12686-12712 
Table 1. Average resistance and surface potential values for P. aeruginosa and MRSA on gold and stainless steel. Increased surface roughness and tip-sample interactions rendered ResiScope unable to retrieve viable measurements of resistance for stainless steel.

\begin{tabular}{|c|c|c|c|c|}
\hline & $\begin{array}{c}\text { Average } \\
\text { Resistance: Au } \\
\text { Substrate }(\mathrm{G} \Omega)\end{array}$ & $\begin{array}{c}\text { Average } \\
\text { Resistance: } \mathrm{SS} \\
\text { Substrate }(\mathrm{G} \Omega)\end{array}$ & $\begin{array}{c}\text { Surface Potential } \\
\text { on Gold } \\
\text { Substrate }(\mathrm{V})\end{array}$ & $\begin{array}{c}\text { Surface Potential } \\
\text { on SS Substrate } \\
(\mathrm{V})\end{array}$ \\
\hline MRSA & $173.4 \pm 16.28$ & --- & $-0.304 \pm 0.05$ & $0.153 \pm 0.06$ \\
\hline P. aeruginosa & $135.4 \pm 25.04$ & --- & $-0.280 \pm 0.09$ & $0.172 \pm 0.02$ \\
\hline
\end{tabular}



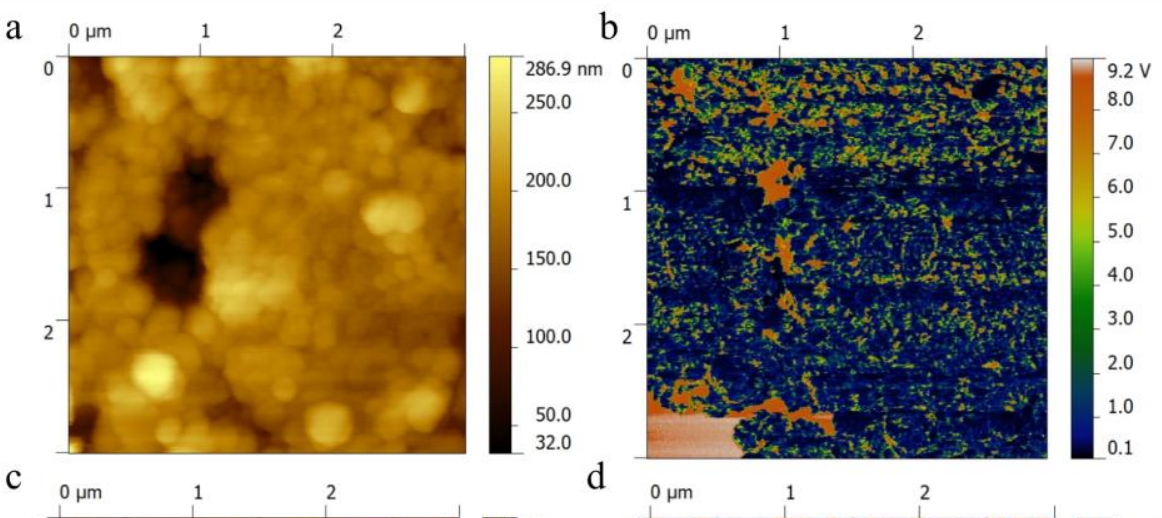

\section{Bare Gold}
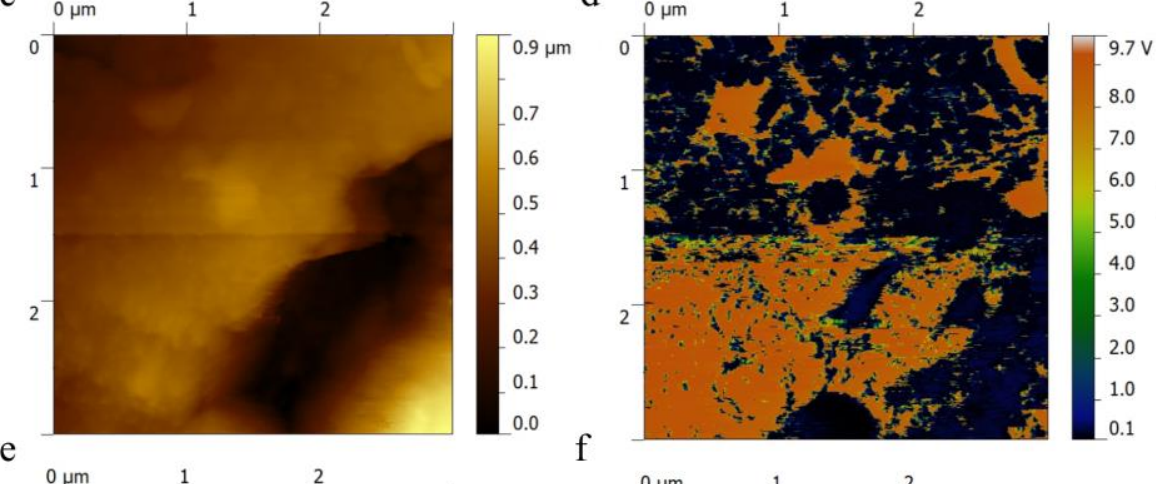

\section{Poly-L-lysine} on Gold
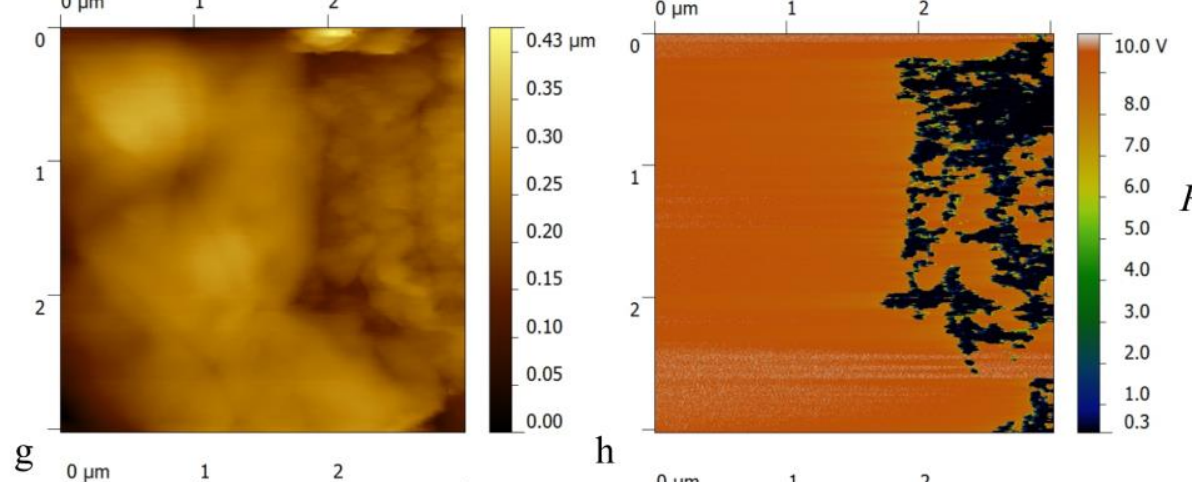

P. aeruginosa

on Gold

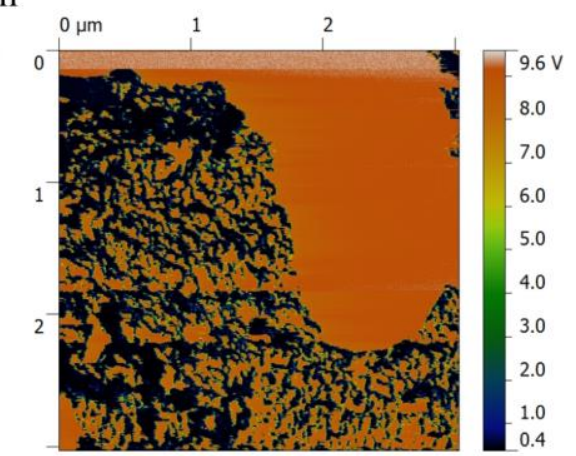

MRSA

on Gold

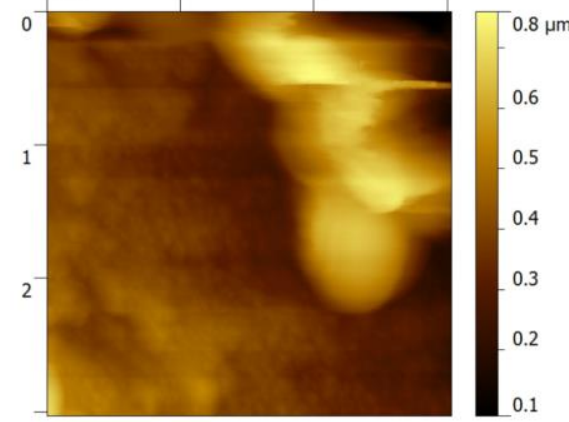

428 Figure 1. Topography and ResiScope resistance images of (a, b) bare gold, (c, d) gold-coated with 429 poly-L-lysine, (e, f) P. aeruginosa and (g, h) MRSA on gold. Application of poly-L-lysine causes 430 a slight increase in highly resistant artifacts on the surface of the gold, but the surface still remains 431 mostly highly conductive. 


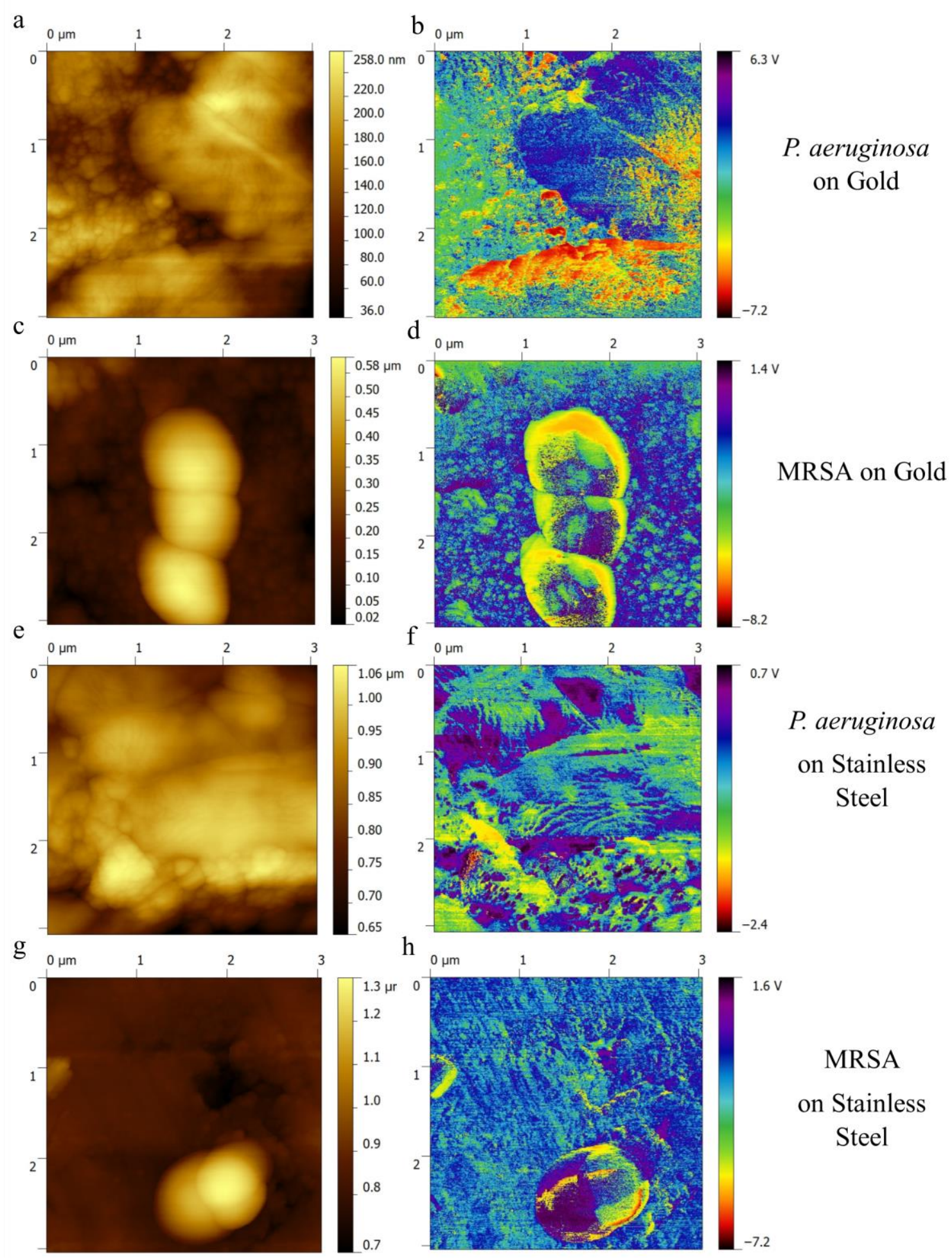

Figure 2. Topography and KPFM (surface potential) images of (a, b) P. aeruginosa on gold, (c, d) MRSA on gold, (e, f) P. aeruginosa on stainless steel and (g, h) MRSA on stainless steel. 436 Surface potential changes from negative for $P$. aeruginosa and MRSA on gold to positive on 437 stainless steel for both bacteria. 
439
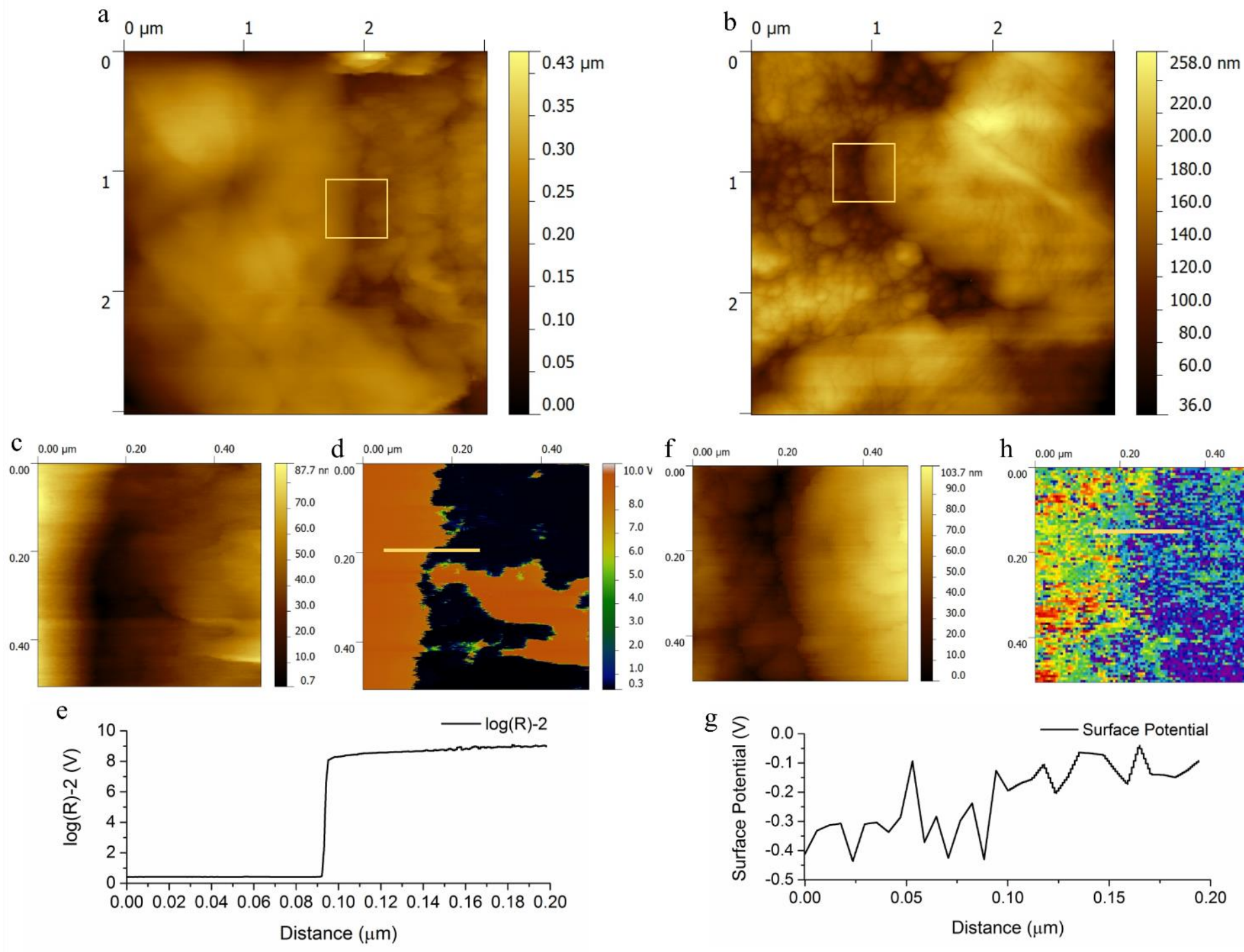

466

467

468 Figure 3. Original $3 \times 3 \mu \mathrm{m}$ zoomed out images of $(\mathrm{a}, \mathrm{b}) P$. aeruginosa on gold substrates. Zoomed $469500 \times 500 \mathrm{~nm}$ topographical scan (c) of $P$. aeruginosa membrane interface with gold substrate and 470 the corresponding resistance image (d). A cross section of the resistance image (e), showing a 471 steep change in resistance. Cropped 500 x $500 \mathrm{~nm}$ topographical scan (f) of P. aeruginosa 472 membrane interface with gold and the corresponding surface potential image $(\mathrm{g})$. Line 473 measurement (h) of surface potential step from gold to bacterial surface.

474

475

476

477 
a

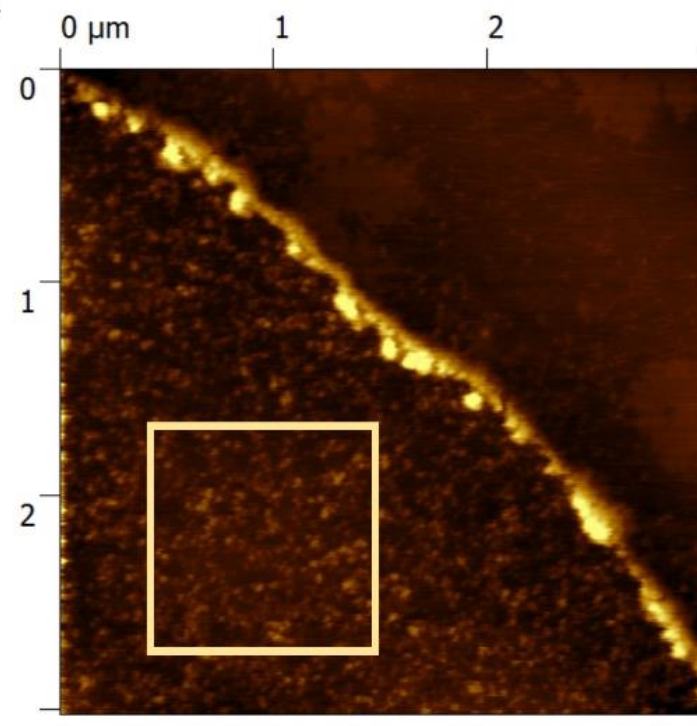

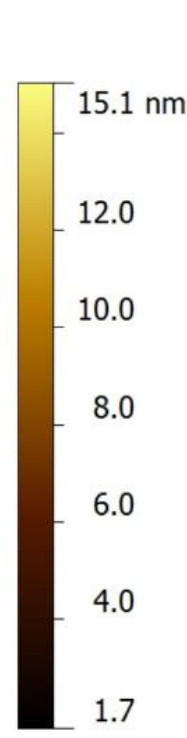

$\mathrm{b}$

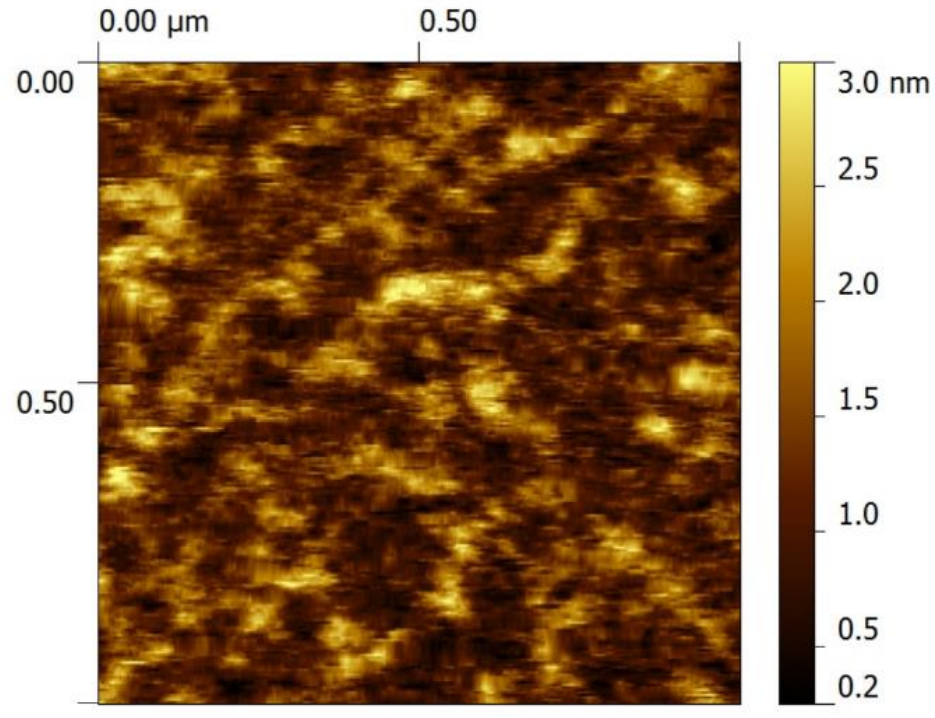

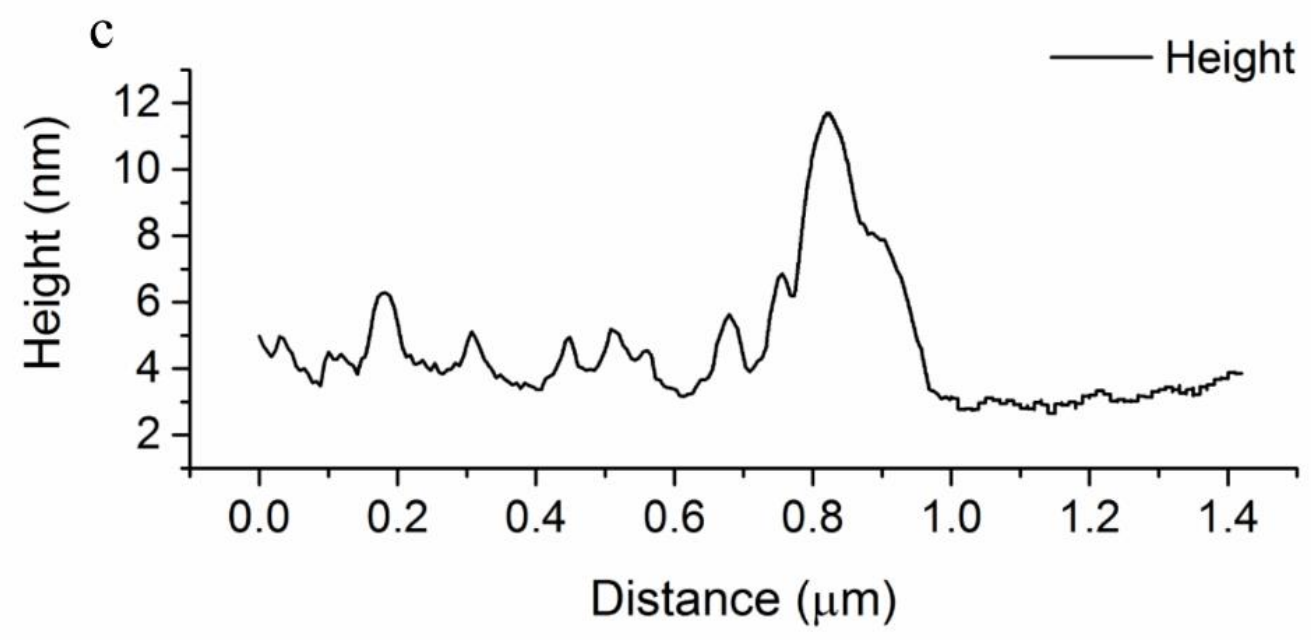

5US

504 Figure 4. Topography images of peptidoglycan particle (a) on Au coated mica surface and (b)

505 zoomed in $3 \times 3 \mu \mathrm{m}$ scan of particle edge. Line scan of (c) peptidoglycan particle at the Au coated

506 mica interface, showing a height of $\sim 40 \mathrm{~nm}$. 


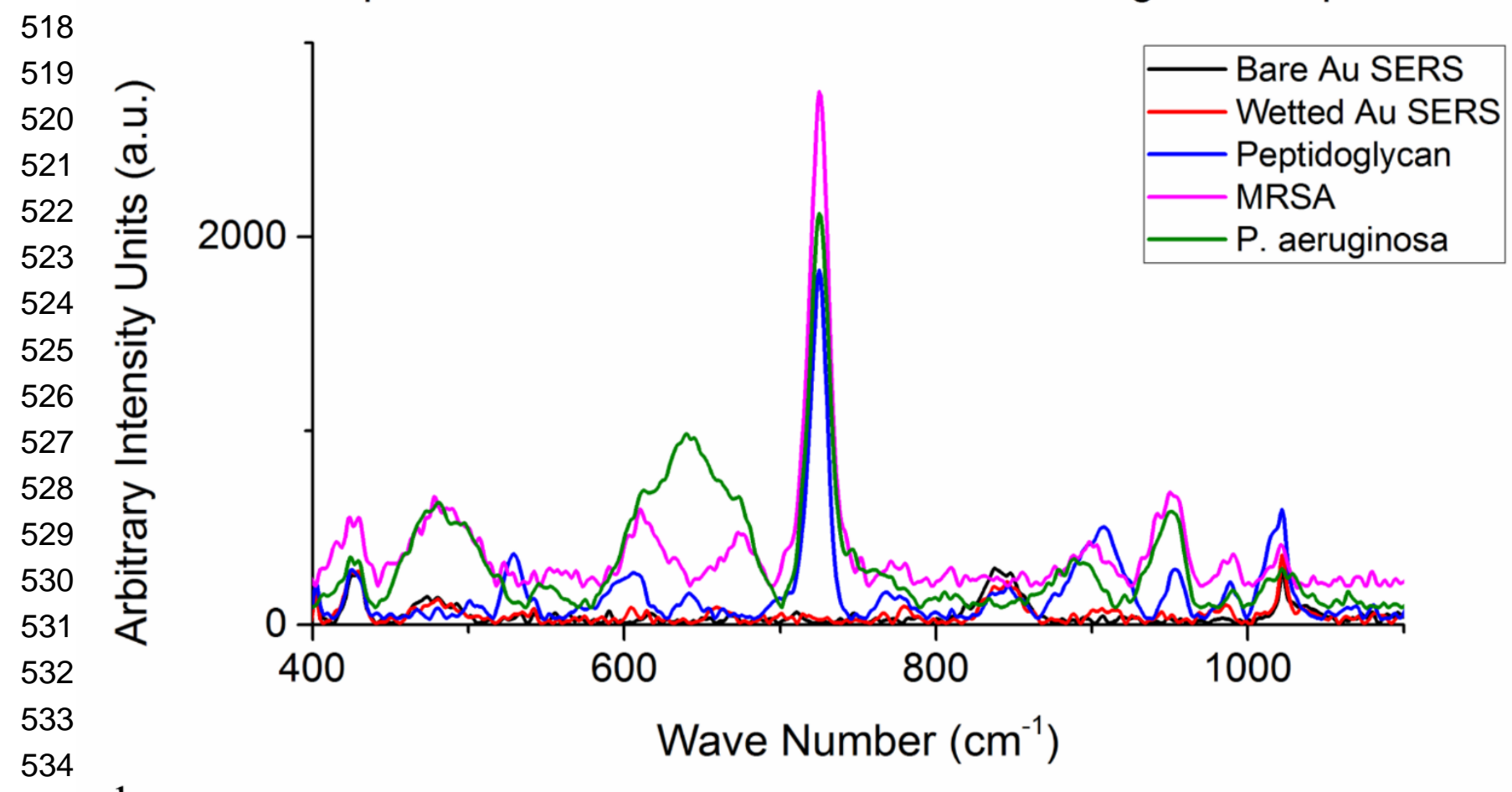

\section{a Comparison of Au-SERS Substrates to Biological Samples}

535

536

537

538

539

540

541

542

543

544

545

546

547

548

549

550

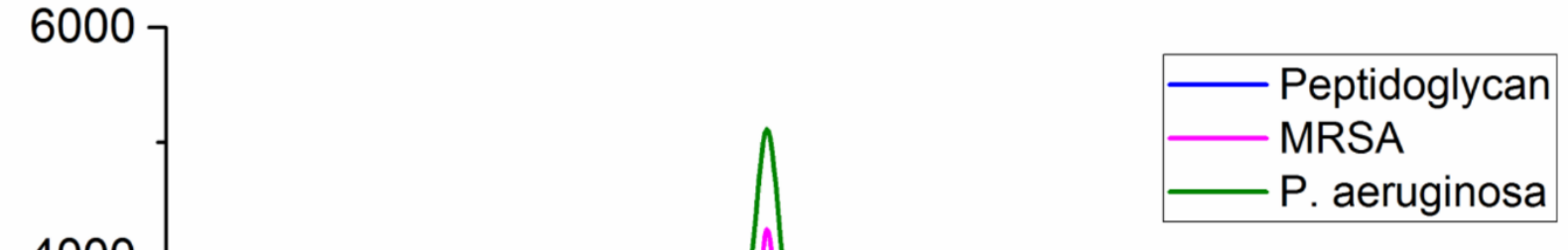

Figure 5. Raman spectra of (a) peptidoglycan in comparison with gold SERS substrate, $P$. aeruginosa and MRSA. Pristine and wetted-then-dried SERS substrates reference spectra show no correlation with peaks of interest. Normalized (b) spectra of peptidoglycan, $P$. aeruginosa and MRSA showing a $\sim 730 \mathrm{~nm}^{-1}$ peak corresponding to glucose rings NAG and NAM molecules, which reside in the peptidoglycan. 


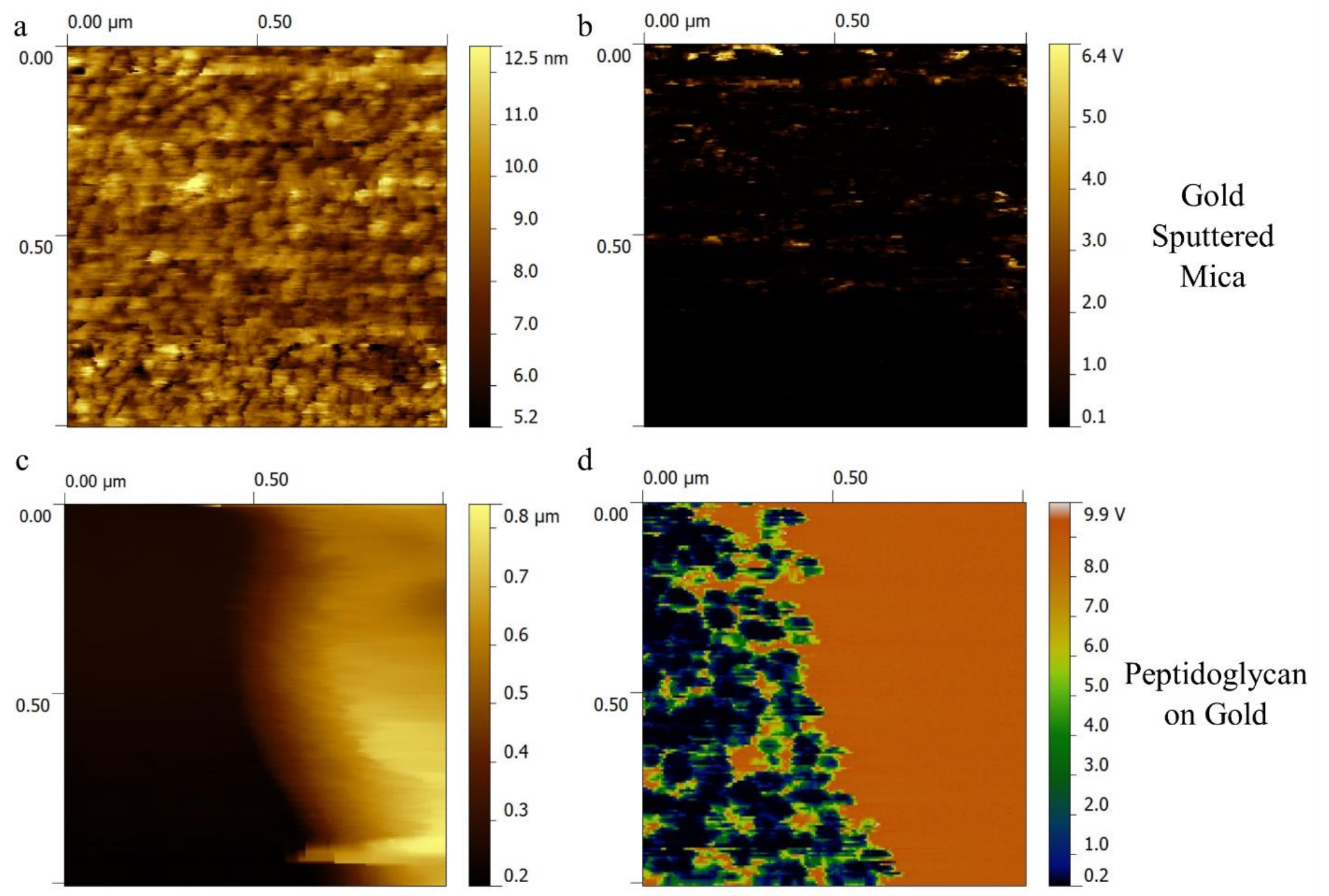

585 Figure 6. Topography and resistance $1 \times 1 \mu \mathrm{m}$ images of (a,b) bare gold and (c,d) peptidoglycan. 586 This peptidoglycan image is from the same peptidoglycan particle as in Figure 4. The average 587 resistance value for peptidoglycan particle on gold surface is $103 \mathrm{G} \Omega$. 